\title{
Optimisation of electrospinning parameter for Poly(L-lactic) acid (PLLA) electrospun nanofiber
}

\author{
Marini Sawawi ${ }^{1}$, S.K Sahari ${ }^{1}$, Nicholas Kuan ${ }^{1}$, Mahshuri Yusof ${ }^{1}$, Magdalene Andew ${ }^{1}$, SF Salleh ${ }^{1}$, Rohana Sapawi ${ }^{1}$ \\ ${ }^{1}$ Faculty of Engineering, Universiti Malaysia Sarawak, 94300 Kota Samarahan,
}

\begin{abstract}
Poly (L-lactic) Acid (PLLA) ia one of the biodegradable polymer that normally used in the biomedical application. In this work it is shown that electrospinning of PLLA electrospun fibre was affected by electrospinning processing parameters such as the solvent systems selection, polymer solution concentration, flow rate and acceleratingvoltage. Optimisation of these parameters is necessary to obtain a beadless and submicron diameter fibre as desired in this research.
\end{abstract}

\section{Introduction}

Poly (L-lactic) Acid (PLLA) is a thermoplastic, semi crystalline polymer. It has become widely used recently due to its biocompatibility and biodegradability [8]. One of the methods to produce PLLA fibre is using electrospinning. Electrospinning has become an oftreported technique for the fabrication of polymeric fibrous membranes that have been used for a variety of applications including membrane technologies, sensors and in particular for tissue engineering as scaffolds that mimic features of the extracellular matrix [3, 16-18, 20, 25] It is a method that makes use of electrical charge with polymer solutions to produce fine filaments. The basic concept dates from 1902 [5] and a series of patents were obtained by Formhals commencing in the 1930s [7] to produce polymer threads from various polymers using such an electrostatic force. This method has subsequently been recognised as a fast and simple process for making continuous, sub-micron to nano-size fibres, when compared with other conventional methods such as drawing, template synthesis, phase separation, and self-assembly. It also has been used to produce various types of fibre materials ranging from natural polymers, synthetic polymers, polymer solutions, polymer melts and biopolymers. One of the attractive aspects of the electrospinning technique is the flexibility of the process and that the morphology of the fibres can be readily varied by changing processing conditions, including producing fibres with solid, porous and coreshell morphologies. Solid and porous fibres can be obtained by changing the processing condition such as types of solvents and humidity whilst the core-shell morphology is obtained through setting up the inner and outer needle during electrospinning [2, 9, 10, 27]. In its most basic form, electrospun webs are produced from a relatively simple process that consists of a pipette to hold a dissolved polymer solution, a high voltage source and a collector. Briefly, the positive electric field induces charges in the polymer solution, and once this charge build-up exceeds that of the surface tension, a Taylor cone is formed [11]. At this critical voltage, a polymer jet erupts from the cone tip, accelerating through the electric field towards the collector. As the jet travels towards the collector, the solvent evaporates and a solidified polymer fibre is collected. There are a number of parameters that influence the morphology of electrospun nanofibres, some of which include: the concentration of polymer solution, applied voltage, solution feed rate, working distance and ambient parameters $[3,15,19,20]$.

Although electrospinning of PLLA polymers has been reported by others in a range of studies $[4,14,20,26]$, it is clear that using a different apparatus and working conditions such as environment will lead to different fibre morphologies, in addition to any changes in processing conditions. In this research, the PLLA polymer was electrospun and optimization of the processing parameters were performed so that the electospun fibres achieve the surface morphology that is beadless and is of a sub-micron diameter size.

\section{Experimental}

Materials. Poly(L-lactide) Acid (PLLA, Inherent viscosity $=0.9-1.2 \mathrm{dL} / \mathrm{g}$ ) was purchased from Lactel, USA. It is in the form of pellets with a brownish, translucent colour. Dodecyl trimethyl ammonium bromide (DTAB), were purchased from Sigma Aldrich, Australia. Chloroform, dimethylformamide (DMF) and acetone from Merck Pty Ltd, Australia. 
Electrospinning.

The PLLA electrospun web was prepared using the solvent systems and electrospinning condition as shown in Table 1. The PLLA-solvent mixture was dissolved using a magnetic stirrer overnight. The solution was then placed in a $10 \mathrm{~mL}$ syringe with the gauged needle. The electrospinning process was conducted using an electrospinner built in the laboratory which used a high voltage supply from Gamma High Voltage Research, Florida, whilst the syringe pump is from Razel Scientific Instruments, Inc. The fibres were collected onto a flat metallic collector with aluminium (Al) foil placed on top. After collection, the non-woven web was stored in a desiccator under vacuum before further use. This procedure was repeated for a range of concentrations, until beadless fibres with a submicron range diameter were obtained.

\section{Characterisation.}

The morphology of the as-spun fibres was characterized using FEI Phenom Desktop SEM. Prior to imaging, gold coated at $1 \mathrm{~nm}$ thickness with a sputter coater (Cressington 208HR, UK).

Table 1 Electrospinning condition

\begin{tabular}{|c|c|c|c|c|c|}
\hline Solvent system & $\begin{array}{l}\text { Concentration } \\
(\%)(\mathrm{w} / \mathrm{v})\end{array}$ & $\begin{array}{l}\text { Feed rate } \\
(\mathrm{ml} / \mathrm{hr})\end{array}$ & $\begin{array}{l}\text { Voltage } \\
(+\mathrm{kV})\end{array}$ & $\begin{array}{c}\text { Needle } \\
\text { size } \\
\text { (G) }\end{array}$ & $\begin{array}{c}\text { Working } \\
\text { Distance } \\
(\mathrm{cm})\end{array}$ \\
\hline chloroform & 6.5 & 2.4 & 20 & 18 & 15 \\
\hline chloroform & 8 & 2.4 & 20 & 18 & 15 \\
\hline chloroform (1mM DTAB) & 8 & 2.4 & 20 & 18 & 15 \\
\hline chloroform (1mM DTAB) & 12 & 2.4 & 20 & 18 & 15 \\
\hline chloroform (1mM DTAB) & 13.7 & 1.6 & 20 & 18 & 15 \\
\hline chloroform (with 1mM DTAB) & 15.3 & 1.6 & 20 & 21 & 23 \\
\hline $\begin{array}{c}\text { chloroform+acetone }(3: 1)(\text { with } 1 \mathrm{mM} \\
\text { DTAB) }\end{array}$ & 13 & 1.2 & 20 & 21 & 23 \\
\hline $\begin{array}{c}\text { chloroform+acetone }(3: 1) \text { (with } 1 \mathrm{mM} \\
\text { DTAB) }\end{array}$ & 13.7 & 1.2 & 15 & 21 & 23 \\
\hline $\begin{array}{c}\text { chloroform+acetone }(3: 1) \text { (with } 1 \mathrm{mM} \\
\text { DTAB) }\end{array}$ & 15 & 1.2 & 15 & 21 & 21 \\
\hline chloroform+acetone (3:1)(with $1 \mathrm{mM}$ & 16 & 1.2 & 25 & 21 & 21 \\
\hline
\end{tabular}

b) $8 \mathrm{wt} / \mathrm{v} \%$

\section{Result and discussion}

The common solvents that have been used to electrospin PLLA include chloroform [22] and a mixture of chloroform and acetone [23]. We initially present results with chloroform alone and Figure 1 shows PLLA electrospun fibres spun from chloroform at $6.5 \mathrm{wt} / \mathrm{v} \%$ and $8 \mathrm{wt} / \mathrm{v} \%$ concentration, with a needle feed rate of 2.4 $\mathrm{mL} / \mathrm{h}$, through an $18 \mathrm{G}$ gauge needle with a $20 \mathrm{kV}$ voltage, and $15 \mathrm{~cm}$ working distance to the collector.

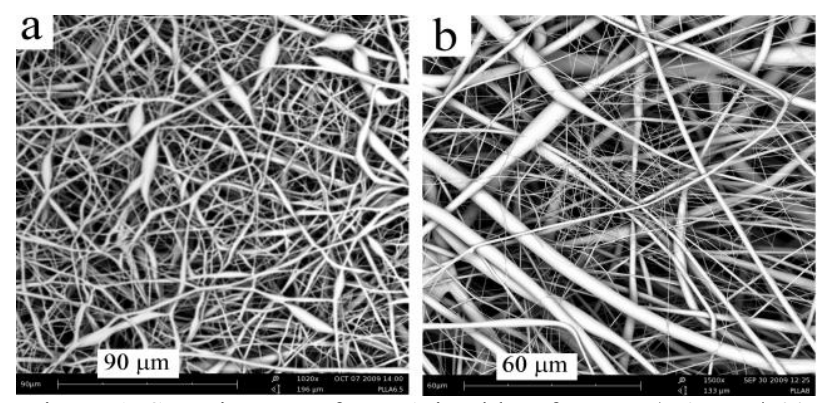

Figure 1 SEM images of PLLA in chloroform at a) $6.5 \mathrm{wt} / \mathrm{v} \%$,
It can be seen in Figure 1 that at $6.5 \mathrm{wt} / \mathrm{v} \%$ polymer concentration, the fibres produced contained elongated beads. Increasing the concentration to $8 \mathrm{wt} / \mathrm{v} \%$ lead to beadless, non uniform fibres, with two distinctive types of fibres' diameter. This bimodal size distribution is due to the secondary jet that erupts from the primary electrospinning jet, producing smaller fibre diameters which result from the fibre-solvent interaction and higher voltage used [20]. It has often been suggested that addition of ionic surfactant can improve the conductivity and reduce the surface tension of the polymer solution $[20,23]$ with the increased conductivity resulting in 
more charge carried by the electrospinning jet, leading to more fibre stretching and thus a thinner fibre [20] The results of incorporation of DTAB are shown in Figure 2, where the PLLA was electrospun in chloroform with 1 $\mathrm{mM}$ DTAB, with the other parameters the same as before for $8 \mathrm{wt} / \mathrm{v} \%, 13.7 \mathrm{wt} / \mathrm{v} \%$ and $15.3 \mathrm{wt} / \mathrm{v} \%$ polymer concentrations.
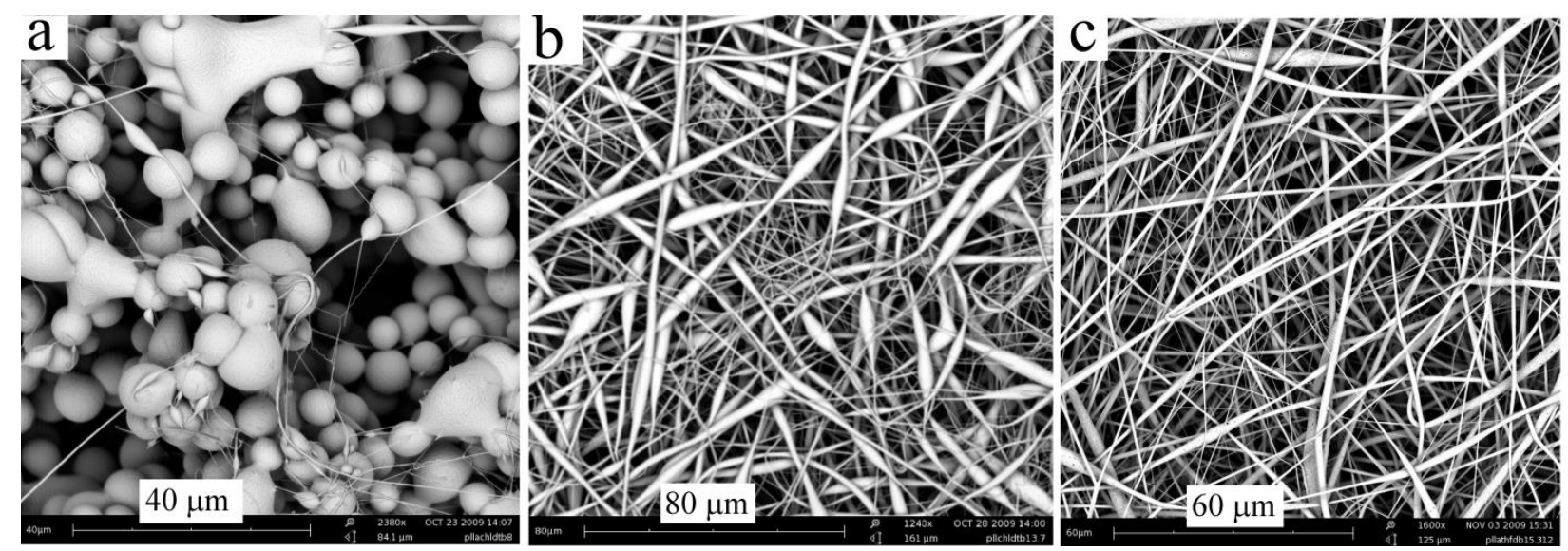

Figure 2 Electrospun PLLA fibres in with chloroform as the solvent, with $1 \mathrm{mM} \mathrm{DTAB}$ at the polymer concentration of a) $8 \mathrm{wt} / \mathrm{v} \%$, b) $13.7 \mathrm{wt} / \mathrm{v} \%$ and c) $15.3 \mathrm{wt} / \mathrm{v} \%$.

It can be seen in Figure 2 that with the addition of the cationic surfactant, DTAB, electrospun fibres in chloroform at $8 \mathrm{wt} / \mathrm{v} \%$ polymer concentration produced fibres with spherical beads, while at the same concentration without DTAB addition, the fibres obtained were beadless (Figure 1b). However, it is important to note that although without addition of DTAB, the fibres showed no beads, the non uniform fibre diameter is undesirable. At $8 \mathrm{wt} / \mathrm{v} \%$ concentration, incorporating of DTAB in chloroform resulted in a difference in charge distribution on the Taylor cone and generated nanofibres with beads [12]. The addition of a cationic surfactant will increase the charge density, leading to a higher conductivity. If the conductivity level is too high, multijets will be produced and lead to fibres with beads $[1,21]$. It was previously shown that without DTAB addition, $8 \mathrm{wt} / \mathrm{v} \%$ results in a bimodal distribution in fibres resulting in distinct fibres diameter due to secondary jets. With the addition of DTAB, an increase in conductivity leads to an unstable jet and results in jet break-ups (electrospraying). It was also found by Kriegel et al [13] that the addition of DTAB reduced the viscosity of $\mathrm{PEO}$ solutions which also might contribute to the beads in our findings. This can be overcome by increasing the concentration at which chain entanglements increase to enable continuous stretching of the jet. This is particularly true when the polymer concentration increased to $12 \mathrm{wt} / \mathrm{v} \%$ (not shown here), and $13.7 \mathrm{wt} / \mathrm{v} \%$ (Figure $2 \mathrm{~b}$ ) where the beads changed from spindle-like shapes to elongated beads. . This shows that by increasing the concentration, the intermolecular force or chain entanglement of the polymer is increased and sufficient for jet stretching. Finally, the optimum fibre morphology for PLLA in chloroform with $1 \mathrm{mM}$ DTAB was obtained at the concentration of $15.3 \mathrm{wt} / \mathrm{v} \%$ with a feed rate of $1.6 \mathrm{~mL} / \mathrm{h}$ , $20 \mathrm{kV}$ accelerating voltage and $15 \mathrm{~cm}$ working distance which obtained average fibre diameter of $790 \pm 390 \mathrm{~nm}$ where no beading was observed although the uniformity of the fibres was still not satisfactory, as shown in the high standard deviation of the fibres diameter.

In order to improve the uniformity and reduced the diameter of the electrospun fibres, PLLA was electrospun with a mixture of chloroform and acetone, with $1 \mathrm{mM}$ DTAB. It has been reported that the addition of acetone to the PLLA in chloroform solution leads to improved continuity of the electrospinning jet [23, 24], likely due to the lower polarity of acetone compared to chloroform, which reduces the interactions between the solvent and polymer molecules [24]. This in turn reduces jet cohesive energy, contributing to a greater ease of spinning and thus more uniform fibre diameters. The electrospinning of PLLA with chloroform and acetone with $1 \mathrm{mM}$ DTAB addition was carried out at the concentration of $13 \mathrm{wt} / \mathrm{v} \%, 13.7 \mathrm{wt} / \mathrm{v} \%, 15 \mathrm{wt} / \mathrm{v} \%$ and $16 \mathrm{wt} / \mathrm{v} \%$, with the other parameters, as stated in Table 1. The result showed that increasing the polymer concentration lead to a larger fibre diameter. This is because the greater concentration leads to a higher solution viscosity and an increased molecular entanglement that leads to high cohesive forces between the polymer molecules, thus reducing the stretching and thinning of the fibres [6]. For the same concentration of $13.7 \mathrm{wt} / \mathrm{v} \%$ PLLA, reducing the accelerating voltage from $15 \mathrm{kV}$ to $10 \mathrm{kV}$ leads to a larger fibre diameter (not shown). This reduced electrostatic field leads to less polymer stretching and fibres with a larger diameter result [20]. For this system, the optimum fibre morphology was obtained at $13.7 \mathrm{wt} / \mathrm{v} \%$ polymer concentration with the feed rate of $1.2 \mathrm{~mL} / \mathrm{h}, 20 \mathrm{kV}$ accelerating voltage, $18 \mathrm{G}$ needle gauge and $16 \mathrm{~cm}$ working distance which obtained the average fibre diameter of $770 \pm 240 \mathrm{~nm}$. The larger fibre average 

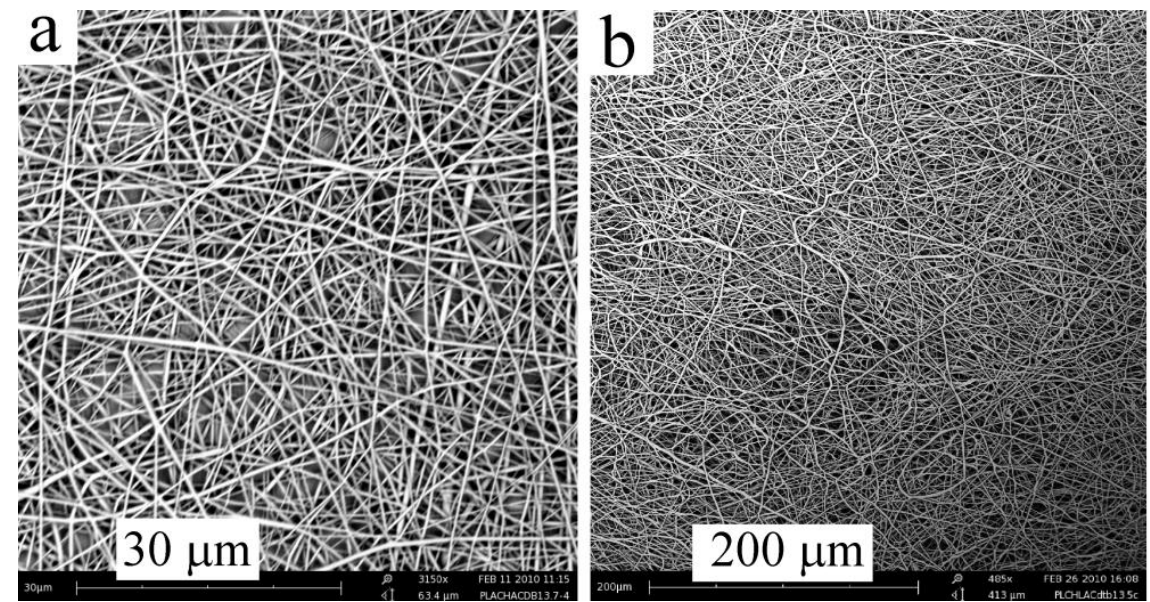

Figure 3 Electrospun PLLA in chloroform:acetone with 1 mMDTAB at a) $13.7 \mathrm{wt} / \mathrm{v} \%$ and b) $16 \mathrm{wt} / \mathrm{v} \%$

Figure 3 demonstrates that the optimum fibre morphology obtained was more uniform for the 13.7 $\mathrm{wt} / \mathrm{v} \%$ PLLA concentration, where the standard deviation of the fibre diameter was about $200 \mathrm{~nm}$. It was thus found that the addition of acetone did improve the electrospinning process of PLLA with the uniformity of the fibres achieved ( lower standard deviation of the fibre diameter showing the distribution of the fibres' diameter is more uniform) with the average fibre diameter within submicron range. Meanwhile, the 16 $w t / v \%$ PLLA concentration showed that the higher concentrations result in higher viscosities that make maintaining flow difficult and the resulting fibres had a greater average fibre diameter and were less uniform.

\section{Conclusion}

From this work, the optimization process of PLLA electrospun fibre were conducted by manipulating the processing parameters such as solvent systems selection, cationic surfactant addition, polymer concentration, polymer solution properties such as viscosity, accelerating voltage and flow rate. For instance, the polymer concentration affects the intermolecular force or chain entanglement that by increasing the concentration, chain entanglement of the polymer is increased and sufficient for jet stretching. Low concentration would lead to less chain entanglement and can cause electrospraying or jet break ups. The addition of DTAB would lead to a change in conductivity and viscosity of the polymer solution. At sufficient concentration and viscosity, this surfactant would produce more uniform fibre compared with no surfactant addition. Reducing the accelerating voltage leads to a larger fibre diameter due to reduced electrostatic field leads to less polymer stretching. By manipulating the parameters, we can produce the fibres at with certain surface morphology and diameter to suit our application.

\section{References}

1. C.J Angammana and H.J Shesha, Industry Applications, IEEE Transactions on. 47(3): p. 11091117, (2011)

2. A. Bazilevsky, A. L. Yarin, and C. M. Megaridis, Langmuir. 23(5): p. 2311-2314, (2007)

3. N Bhardwaj, and C.K Subhas, Biotechnology Advances. 28(3): p. 325-347,(2010)

4. Y.Z Chen et al., Journal of Polymer Science, Part B: Polymer Physics. 47(12): p. 1211-1218, (2009)

5. J.F Cooley Apparatus for electrically dispersing fluids,: US Patents, (1902).

6. J.M Deitzel et al., Polymer. 42(1): p. 261272, (2001)

7. A. Formhals, Process and Apparatus for Preparing artificial threads, US. Patent, Editor (1934).

8. B.Gupta, R. Nilesh, and H.Jöns, Progress in Polymer Science. 32(4): p. 455-482, (2007)

9. C. He et al., Gaojishu Tongxin/Chinese High Technology Letters. 16(9): p. 934-938, (2006)

10. C.L He, Z.M. Huang, and X.J. Han, Journal of Biomedical Materials Research - Part A. 89(1): p. 80-95, (2009)

11. J.H He, et al., Electrospun Nanofibers and Their Applications., Shawbury,UK: iSmithers (2008) 
12. Z.M Huang et al., Composites Science and Technology. 63(15): p. 2223-2253, (2003)

13. C.Kriegel et al., Food Biophysics. 4(3): p. 213-228, (2009)

14. T. Lin et al., Nanotechnology. 15(9): p. 1375, (2004)

15. Y.P Neo et al., Journal of Food Engineering. 109(4): p. 645-651, (2012)

16. D.R Nisbet et al., Journal of Neural Engineering. 4(2): p. 35, (2007)

17. D.R Nisbet et al., Journal of Biomaterials Applications. 24(1): p. 7-29, (2009)

18. D.R Nisbet et al., Biomaterials. 30(27): p. 4573-4580, (2009)

19. N.Okutan et al, Food Hydrocolloids. 39: p. 19-26, (2014)

20. S. Ramakrishna et al., An Introduction to Electrospinning and Nanofibers, Singapore: World Scientific Pub, (2005)

21. C.Wang et al, Macromolecules. 39(22): p. 7662-7672, (2006)

22. Y. You et al., Journal of Applied Polymer Science. 95(2): p. 193-200, (2005)

23. J.Zeng et al., Journal of Controlled Release. 92(3): p. 227-231, (2003)

24. J.Zeng_et al., Journal of Applied Polymer Science. 89(4): p. 1085-1092, (2003)

25. Y.L Zhang et al. Electrospinning process of ethylene-co-vinyl alcohol nanofiber with DMAC/IPA solution system. in Advanced Materials Research.. Trans Tech Publ (2014)

26. Z.Zhou et al., Polymer. 50(13): p. 29993006, (2009)

27. E.Zussman et al., Journal of Polymer Science, Part B: Polymer Physics. 44(10): p. 14821489, (2006) 